PROCEEDINC;S OF: THE.

AMERICAN MATHEMATICAL SOCIETY

Volume 87. Number 3, March 1983

\title{
STABLE HOMOTOPY TYPES OF STUNTED REAL PROJECTIVE SPACES
}

\author{
TEIICHI KOBAYASHI ${ }^{\prime}$
}

\begin{abstract}
The purpose of this note is to improve partly necessary conditions for two stunted real projective spaces to be of the same stable homotopy type given in [4].
\end{abstract}

1. Introduction. Let $R P^{n}$ be the $n$-dimensional real projective space. If $k \geqslant 0$, we have natural inclusion $R P^{n-1} \subset R P^{n+k}$, and denote by $R P_{n}^{n+k}$ the quotient space $R P^{n+k} / R P^{n-1}$. S. Feder, S. Gitler and M. E. Mahowald in [4] have obtained necessary and sufficient conditions for the stunted real projective spaces $R P_{n}^{n+k}$ and $R P_{m}^{m+h}$ to be of the same stable homotopy type for a great number of values of $n, m$ and $k$. Let $\phi(k)$ be the number of integers $s$ such that $0<s \leqslant k$ and $s \equiv 0,1,2$ or 4 $(\bmod 8)$, and let $A_{k}=2^{\phi(k)}$.

THEOREM 1. Let $k$ and $n$ be integers such that $n \equiv 0\left(\bmod A_{k} / 2\right)$. If $n \neq \equiv m$ $\left(\bmod A_{k}\right)$, then the spaces $R P_{n}^{n+k}$ and $R P_{m}^{m+k}$ are not of the same stable homotopy type.

Theorem 1 partly improves Theorem 1.1 in $[4] .^{2}$ For the converse of the result, it is well known (cf. [1-3]) that if $n \equiv m\left(\bmod A_{k}\right)$, then the spaces $R P_{n}^{n+k}$ and $R P_{m}^{m+k}$ are of the same stable homotopy type.

2. Proof of Theorem 1. In case $k \leqslant 8$, we can prove without difficulty that if $R P_{n}^{n+k}$ and $R P_{m}^{m+k}$ are of the same stable homotopy type, then $n \equiv m\left(\bmod A_{k}\right)$, using naturality of the squaring operations.

Consider the case $k>8$. Suppose $m<n$ and there is a homotopy equivalence $f$ : $S^{t} R P_{n}^{n+k} \rightarrow S^{t+n-m} R P_{m}^{m+k}$ for some integer $t \geqslant 0$. Then $n \equiv m\left(\bmod A_{k} / 2\right)$, by Theorem 1.1 in [4] (cf. also [5 and 6]). We may assume $t \equiv 0(\bmod 8)$, and so $t+n-m \equiv 0(\bmod 8)$. Put $t=8 u$ and $t+n-m=8 v$ and let

$$
I^{u}: \widetilde{K O}\left(R P_{n}^{n+k}\right) \rightarrow \widetilde{K O}\left(S^{t} R P_{n}^{n+k}\right), \quad I^{v}: \widetilde{K O}\left(R P_{m . .}^{m+k}\right) \rightarrow \widetilde{K O}\left(S^{x t} R P_{m}^{m+k}\right)
$$

Received by the editors January 27, 1982.

1980 Mathematics Subject Classification. Primary 55P10: Secondary 55N15.

Key words and phrases. Stunted real projective space, stable homotopy type, $K O$-theory, Adams operation.

'The author wishes to express his thanks to the referee for his kind suggestion.

${ }^{2}$ Theorem 1 was known to the authors of [4]. but the result was not published. 
be the isomorphisms defined by the Bott periodicity. If $l \leqq \equiv 0(\bmod 4)$, we have by $[1$, Theorem 7.4],

$$
\widetilde{K O}\left(R P_{l}^{l+k}\right) \cong Z \oplus \widetilde{K O}\left(R P_{l+l}^{l+k}\right) \cong Z \oplus Z_{A_{k}} \text {. }
$$

Denote by $\nu^{(l)}$ and $\lambda^{(l+1)}$ the generators of the first summand and the second summand, respectively. We may put

$$
f^{*} I^{v} \nu^{(m)}=\varepsilon I^{u} \nu^{(n)}+a I^{u} \lambda^{(n+1)}, \quad f^{*} I^{v} \lambda^{(m+1)}=(2 b+\varepsilon) I^{u} \lambda^{(n+1)},
$$

where $a$ and $b$ are some integers and $\varepsilon= \pm 1$. Let $\Psi^{3}$ be the third Adams operation. Then by [1, Theorem 5.1, Corollary 5.3 and Theorem 7.4], we have

$$
\begin{aligned}
\Psi^{3} f^{*} I^{v} \nu^{(m)}= & f^{*} \Psi^{3} I^{v} \nu^{(m)}=3^{4 v} f^{*} I^{v} \Psi^{3} \nu^{(m)} \\
= & 3^{4 v} f^{*} I^{v}\left\{3^{m / 2} \nu^{(m)}+2^{-1}\left(3^{m / 2}-1\right) \lambda^{(m+1)}\right\} \\
= & 3^{4 v}\left\{3^{m / 2}\left(\varepsilon I^{u} \nu^{(n)}+a I^{u} \lambda^{(n+1)}\right)+2^{-1}\left(3^{m / 2}-1\right)(2 b+\varepsilon) I^{u} \lambda^{(n+1)}\right\} \\
= & \varepsilon 3^{4 u+n / 2} I^{u} \nu^{(n)} \\
& +3^{4 u}\left[3^{n / 2} a+3^{(n-m) / 2}\left\{\left(3^{m / 2}-1\right) b+2^{-1}\left(3^{m / 2}-1\right) \varepsilon\right\}\right] I^{u} \lambda^{(n+1)} .
\end{aligned}
$$

On the other hand,

$$
\begin{aligned}
\Psi^{3} f^{*} I^{v} \nu^{(m)} & =\varepsilon \Psi^{3} I^{u} \nu^{(n)}+a \Psi^{3} I^{u} \lambda^{(n+1)}=\varepsilon 3^{4} u I^{u} \Psi^{3} \nu^{(n)}+a 3^{4 u} I^{u} \Psi^{3} \lambda^{(n+1)} \\
& =\varepsilon 3^{4 u} I^{u}\left\{3^{n / 2} \nu^{(n)}+2^{-1}\left(3^{n / 2}-1\right) \lambda^{(n+1)}\right\}+3^{4} u a I^{u} \lambda^{(n+1)} \\
& =\varepsilon 3^{4 u+n / 2} I^{u} \nu^{(n)}+3^{4 u}\left\{2^{-1}\left(3^{n / 2}-1\right) \varepsilon+a\right\} I^{u} \lambda^{(n+1)} .
\end{aligned}
$$

We have, therefore,

$$
3^{n / 2} a+3^{(n-m) / 2}\left\{\left(3^{m / 2}-1\right) b+2^{-1}\left(3^{m / 2}-1\right) \varepsilon\right\} \equiv 2^{-1}\left(3^{n / 2}-1\right) \varepsilon+a
$$

$\left(\bmod A_{k}\right)$,

because the order of the element $I^{u} \lambda^{(n+1)}$ is equal to $A_{k}=2^{\phi(k)}$. Since $n \equiv m \equiv 0$ $\left(\bmod A_{k} / 2\right), 3^{n / 2}-1 \equiv 3^{m / 2}-1 \equiv 0\left(\bmod A_{k}\right)$ by [1, Lemma 8.1]. Hence we obtain $3^{(n-m) / 2}-1 \equiv 0\left(\bmod 2 A_{k}\right)$, and so we get $n-m \equiv 0\left(\bmod A_{k}\right)$ by [1, Lemma 8.1].

3. Remark. Combining Theorem 1 with known results, we have

Corollary 2. Let $k \leqslant 8$, or $k>8$ and $n \equiv 0\left(\bmod A_{k} / 2\right)$. Then $R P_{n}^{n+k}$ and $R P_{m}^{m+k}$ are of the same stable homotopy type if and only if $n \equiv m\left(\bmod A_{k}\right)$.

\section{REFERENCES}

I. J. F. Adams, Vector fields on spheres, Ann. of Math. (2) 75 (1962), 603-632.

2. On the groups $J(X)$. II, Topology 3 (1965), 137-171.

3. M. F. Atiyah, Thom complexes, Proc. London Math. Soc. 11 (1961), 291-310.

4. S. Feder, S. Gitler and M. E. Mahowald, On the stable homotopy type of stunted projective spaces, Bol. Soc. Mat. Mexicana 22 (1977), 1-5.

5. T. Kobayashi, On the odd order nonsingular immersions of real projective spaces. J. Sci. Hiroshima Univ. Ser. A-I 33 (1969), 197-207.

6. T. Kobayashi and M. Sugawara, On stable homotopy types of stunted lens spaces, Hiroshima Math. J. 1 (1971), 287-304

Department of Mathematics, Faculty of Science, Kochi University, Kochi 780, Japan 